\title{
LOG-MAJORIZATIONS FOR THE (SYMPLECTIC) EIGENVALUES OF THE CARTAN BARYCENTER
}

\author{
FUMIO HIAI AND YONGDO LIM
}

\begin{abstract}
In this paper we show that the eigenvalue map and the symplectic eigenvalue map of positive definite matrices are Lipschitz for the Cartan-Hadamard Riemannian metric, and establish log-majorizations for the (symplectic) eigenvalues of the Cartan barycenter of integrable probability Borel measures. This leads a version of Jensen's inequality for geometric integrals of matrix-valued integrable random variables.
\end{abstract}

2010 Mathematics Subject Classification. 15A42, 47A64, 47B65, 47L07

Key words and phrases. Positive definite matrix, Riemannian trace metric, Cartan barycenter, probability measure, (symplectic) eigenvalue, log-majorization.

\section{INTRODUCTION}

Let $\mathbb{S}_{n}$ be the Euclidean space of $n \times n$ real symmetric matrices equipped with the trace inner product $\langle X, Y\rangle=\operatorname{tr}(X Y)$. Let $\mathbb{P}_{n} \subset \mathbb{S}_{n}$ be the open convex cone of real positive definite matrices, which is a smooth Riemannian manifold with the Riemannian trace metric $\langle X, Y\rangle_{A}=\operatorname{tr} A^{-1} X A^{-1} Y$, where $A \in \mathbb{P}_{n}$ and $X, Y \in \mathbb{S}_{n}$. This is an important example of Cartan-Hadamard manifolds, simply connected complete Riemannian manifolds with non-positive sectional curvature (the canonical 2-tensor is non-negative). The Riemannian distance between $A, B \in \mathbb{P}_{n}$ with respect to the above metric is given by $\delta(A, B)=\left\|\log A^{-1 / 2} B A^{-1 / 2}\right\|_{2}$, where $\|X\|_{2}=\left(\operatorname{tr} X^{2}\right)^{1 / 2}$ for $X \in \mathbb{S}_{n}$.

One of recent active research topics on this Riemannian manifold $\mathbb{P}_{n}$ is the Cartan mean (alternatively the Riemannian mean, the Karcher mean)

$$
G\left(A_{1}, \ldots, A_{m}\right):=\underset{X \in \mathbb{P}}{\arg \min } \sum_{j=1}^{m} \delta^{2}\left(A_{j}, X\right),
$$

Date: November 12, 2018. 
where the minimizer exits uniquely. This is a multivariate extension of the twovariable geometric mean $A \# B:=A^{1 / 2}\left(A^{-1 / 2} B A^{-1 / 2}\right)^{1 / 2} A^{1 / 2}$, which is the unique midpoint between $A$ and $B$ for the Riemannian trace metric, and it retains most of its attractive properties; for instances, joint homogeneity, monotonicity, joint concavity, and the arithmetic-geometric-harmonic mean inequalities. It also extends the multivariate geometric mean on $\mathbb{R}_{+}^{n} \subset \mathbb{P}_{n}$, where $\mathbb{R}_{+}=(0, \infty)$, via the embedding into diagonal matrices, $\left(a_{1}, \ldots, a_{n}\right) \mapsto \operatorname{diag}\left(a_{1}, \ldots, a_{n}\right)$.

The Cartan mean extends uniquely to a contractive (with respect to the Wasserstein metric) barycentric map on the Wasserstein space of $L^{1}$-probability measures;

$$
G: \mathcal{P}^{1}\left(\mathbb{P}_{n}\right) \rightarrow \mathbb{P}_{n}
$$

where a probability Borel measure $\mu$ belongs to $\mathcal{P}^{1}\left(\mathbb{P}_{n}\right)$ if $\int_{\mathbb{P}_{n}} \delta(A, X) d \mu(A)<\infty$ for some $X \in \mathbb{P}_{n}$. The Cartan barycenter plays a fundamental role in the theory of integrations (random variables, expectations and variances). Let $(\Omega, \mathbf{P})$ be a probability space and let $L^{1}\left(\Omega ; \mathbb{P}_{n}\right)$ be the space of measurable functions $\varphi: \Omega \rightarrow \mathbb{P}_{n}$ such that $\int_{\Omega} \delta(\varphi(\omega), X) d \mathbf{P}(\omega)<\infty$ for some $X \in \mathbb{P}_{n}$. Then the "geometric" integral of $\varphi \in L^{1}\left(\Omega ; \mathbb{P}_{n}\right)$ is naturally defined as

$$
\int_{\Omega}^{(G)} \varphi(\omega) d \mathbf{P}(\omega):=G\left(\varphi_{*} \mathbf{P}\right) .
$$

Here, we use the notation $\int_{\Omega}^{(G)}$ to avoid the confusion with the usual $\int_{\Omega}$ in the Euclidean (or arithmetic) sense, that is, $\int_{\Omega} \varphi(\omega) d \mathbf{P}(\omega)=\mathcal{A}\left(\varphi_{*} \mathbf{P}\right)$, where $\mathcal{A}: \mathcal{P}^{\infty}\left(\mathbb{P}_{n}\right) \rightarrow$ $\mathbb{P}_{n}$ is the arithmetic barycenter on the space of bounded probability measures.

In this paper we consider the eigenvalue mapping on $\mathbb{P}_{n}$

$$
\lambda: \mathbb{P}_{n} \rightarrow \mathbb{R}_{+}^{n}, \quad \lambda(A)=\left(\lambda_{1}(A), \ldots, \lambda_{n}(A)\right)
$$

ordered as $\lambda_{1}(A) \geq \cdots \geq \lambda_{n}(A)$ counting multiplicities, and the extended symplectic eigenvalue map on $\mathbb{P}_{2 n}$

$$
\widehat{d}: \mathbb{P}_{2 n} \rightarrow \mathbb{R}_{+}^{2 n}, \quad \widehat{d}(A)=\left(\widehat{d}_{1}(A), \widehat{d}_{2}(A), \ldots, \widehat{d}_{2 n}(A)\right) .
$$

The symplectic eigenvalues play an important role in classical Hamiltonian dynamics, in quantum mechanics, in symplectic topology, and in the more recent subject of quantum information; see, e.g., [7, 15]. For every $A \in \mathbb{P}_{2 n}$, Williamson's theorem 
(see [1, 15]) says that there exist a unique diagonal matrix $D=\operatorname{diag}\left(d_{1}, \ldots, d_{n}\right)$ with $0<d_{1} \leq \cdots \leq d_{n}$ and an $M \in \operatorname{Sp}(2 n, \mathbb{R})$, the symplectic Lie group, such that

$$
A=M^{T}\left[\begin{array}{cc}
D & 0 \\
0 & D
\end{array}\right] M
$$

Then, $d(A)=\left(d_{1}(A), \ldots, d_{n}(A)\right):=\left(d_{1}, \ldots, d_{n}\right)$ is called the symplectic eigenvalues of $A$. The extended symplectic eigenvalues $\widehat{d}(A)$ of $A$ is defined by

$$
\widehat{d}_{1}(A)=\widehat{d}_{2}(A)=d_{n}, \ldots, \quad \widehat{d}_{2 n-1}(A)=\widehat{d}_{2 n}(A)=d_{1} .
$$

Our main theorem of the present paper is the following log-majorizations of the (symplectic) eigenvalues of the Cartan barycenter.

Theorem 1.1. The maps $\lambda$ and $\widehat{d}$ are Lipschitz for the Riemannian trace metric. Moreover,

$$
\lambda\left(\int_{\Omega}^{(G)} \varphi(\omega) d \mathbf{P}(\omega)\right) \underset{\log }{\prec} \int_{\Omega}^{(G)} \lambda(\varphi(\omega)) d \mathbf{P}(\omega), \quad \varphi \in L^{1}\left(\Omega ; \mathbb{P}_{n}\right)
$$

and

$$
\widehat{d}\left(\int_{\Omega}^{(G)} \varphi(\omega) d \mathbf{P}(\omega)\right) \underset{\log }{\prec} \int_{\Omega}^{(G)} \widehat{d}(\varphi(\omega)) d \mathbf{P}(\omega), \quad \varphi \in L^{1}\left(\Omega ; \mathbb{P}_{2 n}\right)
$$

Here $\underset{\log }{\prec}$ denotes the log-majorization between positive vectors in $\mathbb{R}_{+}^{n}$; for $a=$ $\left(a_{1}, \cdots, a_{n}\right)$ and $b=\left(b_{1}, \ldots, b_{n}\right)$ in $\mathbb{R}_{+}^{n}$ arranged in decreasing order $a_{1} \geq \cdots \geq a_{n}$ and $b_{1} \geq \cdots \geq b_{n}, a \underset{\log }{\prec} b$ if and only if $\prod_{i=1}^{k} a_{i} \leq \prod_{i=1}^{k} b_{i}$ for $1 \leq k \leq n$ and equality holds for $k=n$. For $A, B \in \mathbb{P}_{n}$ we also write $A \underset{\log }{\prec} B$ if $\lambda(A) \underset{\log }{\prec} \lambda(B)$, which implies that $|\|A\|| \leq|||B|||$ for all unitarily invariant norms $\||\cdot|||$ on the $n \times n$ complex matrices.

The result in the main theorem is a variant of classical Jensen's inequality for integrals and covers those of Bhatia and Karandikar [5] and of Bhatia and Jain [4]:

$$
\lambda\left(G\left(A_{1}, \ldots, A_{m}\right)\right) \underset{\log }{\prec} G\left(\lambda\left(A_{1}\right), \ldots, \lambda\left(A_{m}\right)\right)
$$

and

$$
\widehat{d}\left(G\left(A_{1}, \ldots, A_{m}\right)\right) \underset{\log }{\prec} G\left(\widehat{d}\left(A_{1}\right), \ldots, \widehat{d}\left(A_{m}\right)\right) .
$$




\section{2. (SympleCtic) EigenVAlue MAPPings}

The convex cone $\mathbb{P}_{n}$ is, not only a Riemannian manifold with the Riemannian trace metric, but a Banach Finsler manifold over $\mathbb{S}_{n}$, the Finsler structure being derived from the operator norm $\|X\|_{A}:=\left\|A^{-1 / 2} X A^{-1 / 2}\right\|$ for $A \in \mathbb{P}_{n}$ and $X \in \mathbb{S}_{n}$. The induced metric distance on $\mathbb{P}$ is explicitly given by $d_{T}(A, B)=\left\|\log A^{-1 / 2} B A^{-1 / 2}\right\|$, which is nothing but the Thompson metric

$$
d_{T}(A, B)=\max \{\log M(B / A), \log M(A / B)\},
$$

where $M(B / A):=\inf \{\alpha>0: B \leq \alpha A\}$, the largest eigenvalue of $A^{-1 / 2} B A^{-1 / 2}$. The geometric mean curve $t \mapsto A \#_{t} B:=A^{1 / 2}\left(A^{-1 / 2} B A^{-1 / 2}\right)^{t} A^{1 / 2}$ is a minimal geodesic from $A$ to $B$ for the Thompson metric; see [14, 6]. We observe that

$$
d_{T}(A, B) \leq \delta(A, B) \leq \sqrt{n} d_{T}(A, B)
$$

where $\delta(A, B)=\left\|\log A^{-1 / 2} B A^{-1 / 2}\right\|_{2}$ is the Riemannian distance.

Let $\mathcal{P}^{1}\left(\mathbb{P}_{n}\right)$ be the set of integrable probability Borel measures on $\mathbb{P}_{n}$, i.e., probability Borel measures $\mu$ on $\mathbb{P}_{n}$ such that $\int_{\mathbb{P}_{n}} \delta(A, X) d \mu(A)<\infty$ for some $X \in \mathbb{P}_{n}$. By (2.1) , the Thompson metric leads the same probability measure space $\mathcal{P}^{1}\left(\mathbb{P}_{n}\right)$. That is, for a probability Borel measure $\mu$ on $\mathbb{P}_{n}, \int_{\mathbb{P}_{n}} \delta(A, X) d \mu(A)<\infty$ if and only if $\int_{\mathbb{P}_{n}} d_{T}(A, X) d \mu(A)<\infty$. The Wasserstein metric $\delta^{W}$ on $\mathcal{P}^{1}(\mathbb{P})$ is defined by

$$
\delta^{W}(\mu, \nu):=\inf _{\pi \in \Pi(\mu, \nu)} \int_{\mathbb{P}_{n} \times \mathbb{P}_{n}} \delta(X, Y) d \pi(X, Y)
$$

where $\Pi(\mu, \nu)$ is the set of all couplings for $\mu$ and $\nu$. Similarly we have the Wasserstein distance $d_{T}^{W}$ from the Thompson metric. Both are complete metrics on $\mathcal{P}^{1}\left(\mathbb{P}_{n}\right)$ but they are quite distinctive.

For a general metric space $(X, d)$ one can define $\mathcal{P}^{1}(X)$ to be the set of integrable probability Borel measures whose support has measure 1, and the Wasserstein metric $d^{W}$ on $\mathcal{P}^{1}(X)$ as above. Then the following result appears in [13].

Lemma 2.1. Let $f: X \rightarrow Y$ be a Lipschitz map between complete metric spaces with Lipschitz constant $C$. Then $f_{*}: \mathcal{P}^{1}(X) \rightarrow \mathcal{P}^{1}(Y)$ is $d^{W}$-Lipschitz with Lipschitz constant $C$. 
Note that if $f: \mathbb{P}_{n} \rightarrow \mathbb{P}_{N}$ is a $d_{T}$-Lipschitz map with Lipschitz constant $C$, then it is $\delta$-Lipschitz map with Lipschitz constant $\sqrt{N} C$ by (2.1). It turns out that the Thompson metric is very useful in studying (sub)homogeneous and monotonic mappings. A mapping $f: \mathbb{P}_{n} \rightarrow \mathbb{P}_{N}$ is said to be monotonic if $A \leq B$ implies $f(A) \leq f(B)$, and $f$ is subhomogeneous of degree $r>0$ if $f(t A) \leq t^{r} f(A)$ for all $t \geq 1$ and $A \in \mathbb{P}_{n}$.

Proposition 2.2. Let $f: \mathbb{P}_{n} \rightarrow \mathbb{P}_{N}$ be monotonic and subhomogeneous of degree $r$, then it is $d_{T}$-Lipschitz with Lipschitz constant $r$.

Proof. Let $A, B>0$ and let $\alpha=d(A, B)$. Then $A \leq e^{\alpha} B$ and $B \leq e^{\alpha} A$ by definition of the Thompson metric. Using monotonicity and subhomogeneity of degree $r>0$, we have

$$
f(A) \leq f\left(e^{\alpha} B\right) \leq e^{r \alpha} f(B) \text { and } f(B) \leq f\left(e^{\alpha} A\right) \leq e^{r \alpha} f(A)
$$

and hence $d_{T}(f(A), f(B)) \leq r \alpha=r d_{T}(A, B)$.

Example 2.3. One can see that the eigenvalue map $\lambda: \mathbb{P}_{n} \rightarrow \mathbb{R}_{+}^{n}$ is monotonic and homogeneous of degree 1 . Indeed, this holds true for the $j$ th eigenvalue mappings

$$
\lambda_{i}: \mathbb{P}_{n} \rightarrow \mathbb{R}_{+}, \quad i=1, \ldots, n .
$$

Hence, by Proposition 2.2 and Lemma 2.1, the push-forward mappings $\lambda_{*}: \mathcal{P}^{1}\left(\mathbb{P}_{n}\right) \rightarrow$ $\mathcal{P}^{1}\left(\mathbb{R}_{+}^{n}\right)$ and $\left(\lambda_{i}\right)_{*}: \mathcal{P}^{1}\left(\mathbb{P}_{n}\right) \rightarrow \mathcal{P}^{1}\left(\mathbb{R}_{+}\right)$are $d_{T}^{W}$-Lipschitz with Lipschitz constant 1 . By (2.1) they are also $\delta^{W}$-Lipschitz map with Lipschitz constant $\sqrt{n}$ and 1 , respectively.

In fact, the eigenvalue map is also contractive for the Riemannian trace metric $\delta$.

Proposition 2.4. The eigenvalue map $\lambda: \mathbb{P}_{n} \rightarrow \mathbb{R}_{+}^{n}$ is $\delta$-contractive;

$$
\delta(\lambda(A), \lambda(B)) \leq \delta(A, B), \quad A, B \in \mathbb{P}_{n}
$$

Moreover, $\delta^{W}\left(\lambda_{*} \mu, \lambda_{*} \nu\right) \leq \delta^{W}(\mu, \nu)$ for $\mu, \nu \in \mathcal{P}^{1}\left(\mathbb{P}_{n}\right)$.

Proof. The first assertion follows from the Lidskii-Wielandt theorem (see, e.g., [2, 8]) and the EMI property (exponential metric increasing property, see [3]); for $A, B \in \mathbb{P}_{n}$,

$$
\begin{aligned}
\delta(\lambda(A), \lambda(B)) & =\|\log \lambda(A)-\log \lambda(B)\|_{2}=\|\lambda(\log A)-\lambda(\log B)\|_{2} \\
& \leq\|\log A-\log B\|_{2} \leq \delta(A, B) .
\end{aligned}
$$

The latter follows from Lemma 2.1. 
Next, we consider the symplectic eigenvalue map of $2 n \times 2 n$ real positive definite matrices. Let $\mathbb{M}_{2 n}(\mathbb{R})$ be the $2 n \times 2 n$ real matrices and let $J:=\left[\begin{array}{cc}0 & I \\ -I & 0\end{array}\right]$ so that $J^{T}=J^{-1}=-J$. Let $\operatorname{Sp}(2 n, \mathbb{R})$ denote the group of real symplectic matrices, i.e.,

$$
\operatorname{Sp}(2 n, \mathbb{R}):=\left\{M \in \mathbb{M}_{2 n}(\mathbb{R}): M^{T} J M=J\right\} .
$$

It is straightforward to see that the extended symplectic eigenvalue mapping (1.1)

$$
\widehat{d}: \mathbb{P}_{2 n} \rightarrow \mathbb{R}_{+}^{2 n}
$$

is homogeneous of degree 1 . The following shows that it is monotonic.

Theorem 2.5. The extended symplectic eigenvalue map $\widehat{d}$ is monotonic, i.e., for $A, B \in \mathbb{P}_{2 n}, A \leq B$ implies $\widehat{d}(A) \leq \widehat{d}(B)$. Furthermore, for $A, B \in \mathbb{P}_{2 n}$,

$$
d_{T}(\widehat{d}(A), \widehat{d}(B)) \leq d_{T}(A, B) \quad \text { and } \quad \delta(\widehat{d}(A), \widehat{d}(B)) \leq \sqrt{2 n} \delta(A, B) .
$$

Proof. We first show that

$$
\widehat{d}(A)=\lambda^{1 / 2}\left(A^{1 / 2} J^{T} A J A^{1 / 2}\right), \quad A \in \mathbb{P}_{2 n} .
$$

Let $A \in \mathbb{P}_{2 n}$. By definition of the symplectic eigenvalues of $A$, there exist a diagonal matrix $D=\operatorname{diag}\left(d_{1}, \ldots, d_{n}\right)$ with $0<d_{1} \leq \cdots \leq d_{n}$ and an $M \in \operatorname{Sp}(2 n, \mathbb{R})$ such that $A=M^{T}\left[\begin{array}{cc}D & 0 \\ 0 & D\end{array}\right] M$. Set

$$
Q:=\left[\begin{array}{cc}
D^{1 / 2} & 0 \\
0 & D^{1 / 2}
\end{array}\right] M A^{-1 / 2},
$$

which is a $2 n \times 2 n$ orthogonal matrix as

$$
Q^{T} Q=A^{-1 / 2} M^{T}\left[\begin{array}{cc}
D & 0 \\
0 & D
\end{array}\right] M A^{-1 / 2}=A^{-1 / 2} A A^{-1 / 2}=I .
$$

Since $M \in \operatorname{Sp}(2 n, \mathbb{R})$ implies $M^{T} \in \operatorname{Sp}(2 n, \mathbb{R})$ and hence $M J M^{T}=J$, we have

$$
\begin{aligned}
Q A^{1 / 2} J A^{1 / 2} Q^{T} & =\left[\begin{array}{cc}
D^{1 / 2} & 0 \\
0 & D^{1 / 2}
\end{array}\right] M J M^{T}\left[\begin{array}{cc}
D^{1 / 2} & 0 \\
0 & D^{1 / 2}
\end{array}\right] \\
& =\left[\begin{array}{cc}
D^{1 / 2} & 0 \\
0 & D^{1 / 2}
\end{array}\right] J\left[\begin{array}{cc}
D^{1 / 2} & 0 \\
0 & D^{1 / 2}
\end{array}\right]=\left[\begin{array}{cc}
0 & D \\
-D & 0
\end{array}\right] .
\end{aligned}
$$


This implies that the eigenvalues of the Hermitian $2 n \times 2 n$ matrix $A^{1 / 2}(i J) A^{1 / 2}$ is given as

$$
\lambda\left(A^{1 / 2}(i J) A^{1 / 2}\right)=\lambda\left(\left[\begin{array}{cc}
0 & i D \\
-i D & 0
\end{array}\right]\right)=\left(d_{n}, \ldots, d_{1},-d_{1}, \ldots,-d_{n}\right) .
$$

Therefore,

$$
\begin{aligned}
\lambda^{1 / 2}\left(A^{1 / 2} J^{T} A J A^{1 / 2}\right) & =\lambda\left(\left|A^{1 / 2}(i J) A^{1 / 2}\right|\right) \\
& =\left(d_{n}, d_{n}, d_{n-1}, d_{n-1}, \ldots, d_{1}, d_{1}\right)=\widehat{d}(A) .
\end{aligned}
$$

Next, let $A, B \in \mathbb{P}_{2 n}$ with $A \leq B$. It follows from (2.2) that

$$
\begin{aligned}
\widehat{d}(A) & =\lambda^{1 / 2}\left(A^{1 / 2} J^{T} A J A^{1 / 2}\right) \leq \lambda^{1 / 2}\left(A^{1 / 2} J^{T} B J A^{1 / 2}\right) \\
& =\lambda^{1 / 2}\left(B^{1 / 2} J A J^{T} B^{1 / 2}\right) \leq \lambda^{1 / 2}\left(B^{1 / 2} J B J^{T} B^{1 / 2}\right)=\widehat{d}(B) .
\end{aligned}
$$

The remaining part of proof follows from Proposition 2.2 and (2.1).

By Theorem 2.5 and Lemma 2.1, the push-forward map $\widehat{d}_{*}: \mathcal{P}^{1}\left(\mathbb{P}_{2 n}\right) \rightarrow \mathcal{P}^{1}\left(\mathbb{R}_{+}^{2 n}\right)$ is $d_{T}^{W}$-Lipschitz with Lipschitz constant 1 and is also $\delta^{W}$-Lipschitz with Lipschitz constant $\sqrt{2 n}$. Since $\widehat{d}_{i}$ is monotonic and hence is $d_{T^{-}}$Lipschitz, $\left(\widehat{d}_{i}\right)_{*}: \mathcal{P}^{1}\left(\mathbb{P}_{2 n}\right) \rightarrow$ $\mathcal{P}^{1}\left(\mathbb{R}_{+}\right)$is $d_{T}^{W}$-Lipschitz by Lemma 2.1 again.

\section{Cartan Barycenters}

For $\mu \in \mathcal{P}^{1}\left(\mathbb{P}_{n}\right)$, the Cartan barycenter $G(\mu) \in \mathbb{P}_{n}$ is defined as the unique minimizer

$$
G(\mu)=\underset{Z \in \mathbb{P}_{m}}{\arg \min } \int_{\mathbb{P}_{m}}\left[\delta^{2}(Z, X)-\delta^{2}(Y, X)\right] d \mu(X),
$$

independently of the choice of a fixed $Y \in \mathbb{P}_{n}$ (see [16]). Also, the Cartan barycenter is characterized via the Karcher equation (the gradient zero equation) [10] as

$$
X=G(\mu) \Longleftrightarrow \int_{\mathbb{P}} \log X^{-1 / 2} A X^{-1 / 2} d \mu(A)=0 .
$$

An important fact called the fundamental contraction property in [16] (also [10, Theorem 2.3]) is that the Cartan barycenter $G: \mathcal{P}^{1}\left(\mathbb{P}_{n}\right) \rightarrow \mathbb{P}_{n}$ is a Lipschitz map with Lipschitz constant 1; namely, for every $\mu, \nu \in \mathcal{P}^{1}\left(\mathbb{P}_{n}\right)$,

$$
\delta(G(\mu), G(\nu)) \leq \delta^{W}(\mu, \nu)
$$


This contraction property also holds for the Thompson metric [13].

Example 3.1. In the one-dimensional case on $\mathbb{P}_{1}=(0, \infty)=\mathbb{R}_{+}$, we find by a direct computation that for every $\mu \in \mathcal{P}^{1}\left(\mathbb{R}_{+}\right)$,

$$
G(\mu)=\exp \int_{\mathbb{R}_{+}} \log x d \mu(x) .
$$

Similarly, the Cartan barycenter on the product space $\mathbb{R}_{+}^{n}$ is given by

$$
G(\mu)=\exp \int_{\mathbb{R}_{+}^{n}} \log x d \mu(x), \quad \mu \in \mathcal{P}^{1}\left(\mathbb{R}_{+}^{n}\right) .
$$

Here, $\log : \mathbb{R}_{+}^{n} \rightarrow \mathbb{R}^{n}$ is the usual logarithm componentwise on the product space $\mathbb{R}_{+}^{n}$. This coincides with the restriction of the Cartan barycenter $G: \mathcal{P}^{1}\left(\mathbb{P}_{n}\right) \rightarrow \mathbb{P}_{n}$ to $\mathcal{P}^{1}\left(\mathbb{D}_{n}\right)$, where $\mathbb{D}_{n}$ is the set of all diagonal matrices in $\mathbb{P}_{n}$.

We have an explicit formula of $G\left(\lambda_{*} \mu\right)$ for $\mu \in \mathcal{P}^{1}\left(\mathbb{P}_{n}\right)$;

$$
\begin{aligned}
G\left(\lambda_{*} \mu\right) & =\exp \int_{\mathbb{R}_{+}^{n}} \log x d\left(\lambda_{*} \mu\right)(x)=\exp \int_{\mathbb{P}_{n}} \log \lambda(A) d \mu(A) \\
& =\left(\exp \int_{\mathbb{P}_{n}} \log \lambda_{1}(A) d \mu(A), \ldots, \exp \int_{\mathbb{P}_{n}} \log \lambda_{n}(A) d \mu(A)\right) \\
& =\left(\exp \int_{\mathbb{R}_{+}} \log x d\left(\lambda_{1}\right)_{*} \mu(x), \ldots, \exp \int_{\mathbb{R}_{+}} \log x d\left(\lambda_{n}\right)_{*} \mu(x)\right) \\
& =\left(G\left(\left(\lambda_{1}\right)_{*} \mu\right), \ldots, G\left(\left(\lambda_{n}\right)_{*} \mu\right)\right),
\end{aligned}
$$

where in the last equality the map $\left(\lambda_{i}\right)_{*}: \mathcal{P}^{1}\left(\mathbb{P}_{n}\right) \rightarrow \mathcal{P}^{1}\left(\mathbb{R}_{+}\right)$is well-defined by Example 2.3.

Note that for $\mu=(1 / m) \sum_{j=1}^{m} \delta_{A_{j}}$,

$$
\lambda_{*} \mu=\frac{1}{m} \sum_{j=1}^{m} \delta_{\lambda\left(A_{j}\right)} \quad \text { and } \quad\left(\lambda_{i}\right)_{*} \mu=\frac{1}{m} \sum_{j=1}^{m} \delta_{\lambda_{i}\left(A_{j}\right)} .
$$

We have proved the following

Proposition 3.2. For $\mu \in \mathcal{P}^{1}\left(\mathbb{P}_{n}\right)$, we have

$$
G\left(\lambda_{*} \mu\right)=\left(G\left(\left(\lambda_{1}\right)_{*} \mu\right), \ldots, G\left(\left(\lambda_{n}\right)_{*} \mu\right)\right) .
$$


In particular, for $\mu=(1 / m) \sum_{j=1}^{m} \delta_{A_{j}}$,

$$
G\left(\lambda_{*} \mu\right)=G\left(\lambda\left(A_{1}\right), \ldots, \lambda\left(A_{n}\right)\right)=\left(\left[\prod_{j=1}^{m} \lambda_{1}\left(A_{j}\right)\right]^{\frac{1}{m}}, \ldots,\left[\prod_{j=1}^{m} \lambda_{n}\left(A_{j}\right)\right]^{\frac{1}{m}}\right) .
$$

\section{Log-Majorizations FOR PROBABILITy MEASURES}

We have the following diagram involving the eigenvalue map and the Cartan barycenter:

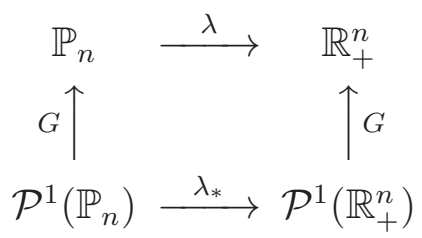

The diagram does not commute, but finding a relationship between $\lambda \circ G$ and $G \circ \lambda_{*}$ seems very interesting. We establish a log-majorization between them, as well as a similar log-majorization for the extended symplectic eigenvalues:

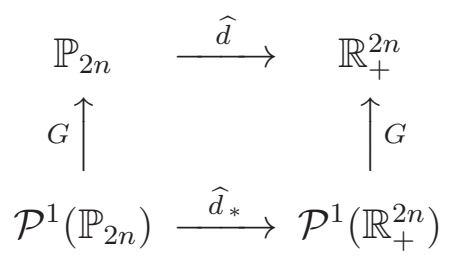

For $0<r<1$ and $\mu \in \mathcal{P}^{1}\left(\mathbb{P}_{n}\right)$, let $\mu^{r}$ denote the push-forward of $\mu$ by the power map $X \mapsto X^{r}$. Indeed, the power map is a strict contraction for the Riemannian trace metric (also for the Thompson metric), as immediately seen from the log-majorization $\lambda\left(A^{-r / 2} B^{r} A^{-r / 2}\right) \underset{\log }{\prec} \lambda^{r}\left(A^{-1 / 2} B A^{-1 / 2}\right), A, B \in \mathbb{P}_{n}$; see [3, p. 229]. Hence the pushforward map $\mu \mapsto \mu^{r}$ is a strict contraction from $\mathcal{P}^{1}\left(\mathbb{P}_{n}\right)$ into itself.

Let $\mathcal{P}_{0}\left(\mathbb{P}_{n}\right)$ be the set of all finitely supported uniform measures on $\mathbb{P}_{n}$, i.e., measures of the form $\mu=(1 / m) \sum_{j=1}^{m} \delta_{A_{j}}, m \in \mathbb{N}$, where $\delta_{A}$ is the point measure of mass 1 at $A \in \mathbb{P}_{n}$. We note that $\mathcal{P}_{0}\left(\mathbb{P}_{n}\right)$ is dense in the Wasserstein space $\mathcal{P}^{1}\left(\mathbb{P}_{n}\right)$ equipped with either $\delta^{W}$ or $d_{T}^{W}$.

Let $\mathcal{P}^{1}\left(\mathbb{S}_{n}\right)$ be the set of probability Borel measures on the Euclidean space $\mathbb{S}_{n}$ with finite first moment, i.e., $\int_{\mathbb{S}_{n}}\|X\|_{2} d \mu(X)<\infty$. For each $\mu \in \mathcal{P}^{1}\left(\mathbb{S}_{n}\right)$, the identity map on $\mathbb{S}_{n}$ is Bochner $\mu$-integrable and $\mathcal{A}(\mu)=\int_{\mathbb{S}_{n}} X d \mu(X)$ is the arithmetic mean of $\mu$. Since the logarithm map log $: \mathbb{P}_{n} \rightarrow \mathbb{S}_{n}$ satisfies $\delta(X, I)=\|\log X\|_{2}$, the pushforward map $\log _{*}$ carries $\mathcal{P}^{1}\left(\mathbb{P}_{n}\right)$ into $\mathcal{P}^{1}\left(\mathbb{S}_{n}\right)$. In fact, the EMI property (exponential 
metric increasing property) implies that $\log _{*}: \mathcal{P}^{1}\left(\mathbb{P}_{n}\right) \rightarrow \mathcal{P}^{1}\left(\mathbb{S}_{n}\right)$ is Lipschitz with Lipschitz constant 1. This shows that the integral $\int_{\mathbb{P}_{n}} \log A d \mu(A) \in \mathbb{S}_{n}$ exists for every $\mu \in \mathcal{P}^{1}\left(\mathbb{P}_{n}\right)$. Moreover, similarly to Proposition 2.4, the push-forward $\lambda_{*}$ : $\mathcal{P}^{1}\left(\mathbb{S}_{n}\right) \rightarrow \mathcal{P}^{1}\left(\mathbb{R}^{n}\right)$ of the eigenvalue map $\lambda: \mathbb{S}_{n} \rightarrow \mathbb{R}^{n}$ is Lipschitz with Lipschitz constant 1 .

Theorem 4.1. We have

$$
\lambda(G(\mu)) \underset{\log }{\prec} \lambda^{\frac{1}{r}}\left(G\left(\mu^{r}\right)\right) \underset{\log }{\prec} \lambda\left(\exp \int_{\mathbb{P}_{n}} \log A d \mu(A)\right) \underset{\log }{\prec} G\left(\lambda_{*} \mu\right)
$$

for every $0<r<1$ and $\mu \in \mathcal{P}^{1}\left(\mathbb{P}_{n}\right)$.

Proof. Let $\mu \in \mathcal{P}^{1}\left(\mathbb{P}_{n}\right)$. The first log-majorization follows from the log-majorization of the Cartan barycenter appearing in [10]

$$
G(\mu) \underset{\log }{\prec} G\left(\mu^{r}\right)^{\frac{1}{r}} \underset{\log }{\prec} G\left(\mu^{s}\right)^{\frac{1}{s}}, \quad 0<s \leq r<1 .
$$

As $s \searrow 0$ the Lie-Trotter formula [1]

$$
\lim _{s \rightarrow 0} G\left(\mu^{s}\right)^{\frac{1}{s}}=\exp \int_{\mathbb{P}_{m}} \log A d \mu(A)
$$

gives

$$
G(\mu) \underset{\log }{\prec} \exp \int_{\mathbb{P}_{m}} \log A d \mu(A)
$$

so that

$$
\log \lambda(G(\mu)) \prec \lambda\left(\int_{\mathbb{P}_{m}} \log A d \mu(A)\right) .
$$

For any $\mu \in \mathcal{P}_{0}\left(\mathbb{P}_{n}\right)$, the Ky Fan majorization (see, e.g., [2, 8]) yields

$$
\lambda\left(\int_{\mathbb{P}_{n}} \log A d \mu(A)\right) \prec \int_{\mathbb{P}_{m}} \lambda(\log A) d \mu(A)=\int_{\mathbb{P}_{n}} \log \lambda(A) d \mu(A) .
$$

As mentioned above the theorem, note that $\log _{*}: \mathcal{P}^{1}\left(\mathbb{P}_{n}\right) \rightarrow \mathcal{P}^{1}\left(\mathbb{S}_{n}\right)$ and $\lambda_{*}$ : $\mathcal{P}^{1}\left(\mathbb{S}_{n}\right) \rightarrow \mathcal{P}^{1}\left(\mathbb{R}^{n}\right)$ are Lipschitz. Hence, by density of $\mathcal{P}_{0}\left(\mathbb{P}_{n}\right)$ in the Wasserstein space $\mathcal{P}^{1}\left(\mathbb{P}_{n}\right)$, the preceding majorization holds for any $\mu \in \mathcal{P}^{1}\left(\mathbb{P}_{n}\right)$. Therefore,

$$
\lambda\left(\exp \int_{\mathbb{P}_{n}} \log A d \mu(A)\right) \underset{\log }{\prec} \exp \int_{\mathbb{P}_{n}} \log \lambda(A) d \mu(A)=G\left(\lambda_{*} \mu\right) .
$$


Applying a measure $\mu=(1 / m) \sum_{j=1}^{m} \delta_{A_{j}} \in \mathcal{P}_{0}\left(\mathbb{P}_{n}\right)$ to (4.1) yields

$$
\begin{aligned}
\lambda\left(G\left(A_{1}, \ldots, A_{m}\right)\right) & \underset{\log }{\prec} \lambda^{\frac{1}{r}}\left(G\left(A_{1}^{r}, \ldots, A_{n}^{r}\right)\right) \underset{\log }{\prec} \lambda\left(\exp \left(\frac{1}{m} \sum_{j=1}^{m} \log A_{j}\right)\right) \\
& \prec G\left(\lambda\left(A_{1}\right), \ldots, \lambda\left(A_{n}\right)\right) \\
& =\left(\left[\prod_{j=1}^{m} \lambda_{1}\left(A_{j}\right)\right]^{\frac{1}{m}}, \ldots,\left[\prod_{j=1}^{m} \lambda_{n}\left(A_{j}\right)\right]^{\frac{1}{m}}\right)
\end{aligned}
$$

thanks to Proposition 3.2

Remark 4.2. Although we confine ourselves in this paper to the real positive definite matrices, the results for the eigenvalue map hold true when $\mathbb{P}_{n}$ is the $n \times n$ complex positive definite matrices.

Finally we consider the extended symplectic eigenvalue map $\widehat{d}$.

Theorem 4.3. For every $\mu \in \mathcal{P}^{1}\left(\mathbb{P}_{2 n}\right)$,

$$
\widehat{d}^{\frac{1}{r}}\left(G\left(\mu^{r}\right)\right) \underset{\log }{\prec} G\left(\widehat{d}_{*} \mu\right), \quad 0<r \leq 1 .
$$

To prove the theorem, we first settle the case where $\mu \in \mathcal{P}_{0}\left(\mathbb{P}_{2 n}\right)$. For this we consider slightly more generally the Cartan mean (or the Karcher mean) $G_{w}\left(A_{1}, \ldots, A_{m}\right)$ of $A_{1}, \ldots, A_{m} \in \mathbb{P}_{2 n}$ with a weight $w=\left(w_{1}, \ldots, w_{m}\right), w_{j} \geq 0$ and $\sum_{j=1}^{m} w_{j}=1$.

Lemma 4.4. For every $A, \ldots, A_{m} \in \mathbb{P}_{2 n}$,

$$
\widehat{d}^{\frac{1}{r}}\left(G_{w}\left(A_{1}^{r}, \ldots, A_{m}^{r}\right)\right) \underset{\log }{\prec} G_{w}\left(\widehat{d}\left(A_{1}\right), \ldots, \widehat{d}\left(A_{m}\right)\right), \quad 0<r \leq 1 .
$$

Proof. When $r=1$ this was shown in [4], but the proof below is rather different from that in [4]. First, note that for every $A \in \mathbb{P}_{2 n}$ and $\alpha>0$,

$$
\widehat{d}_{1}(A) \leq \alpha \quad J^{T} A J \leq \alpha^{2} A^{-1} .
$$

Indeed, this is immediately seen from (2.2) since

$$
\lambda^{1 / 2}\left(A^{1 / 2} J^{T} A J A^{1 / 2}\right) \leq \alpha \quad \Longleftrightarrow \quad J^{T} A J \leq \alpha^{2} A^{-1} .
$$


Now for $j=1, \ldots, m$ let $\alpha_{j}:=\widehat{d}_{1}\left(A_{j}\right)$; then $J^{T} A_{j} J \leq \alpha_{j}^{2} A_{j}^{-1}$ by (4.3). Since $0<r \leq$ $1, J^{T} A_{j}^{r} J \leq \alpha_{j}^{2 r} A_{j}^{-r}$ for $j=1, \ldots, m$. By congruence invariance, monotonicity, joint homogeneity and self-duality of $G_{w}$ (see [12]) we have

$$
\begin{aligned}
J^{T} G_{w}\left(A_{1}^{r}, \ldots, A_{m}^{r}\right) J & =G_{\omega}\left(J^{T} A_{1}^{r} J, \ldots, J^{T} A_{m}^{r} J\right) \\
& \leq G_{w}\left(\alpha_{1}^{2 r} A_{1}^{-r}, \ldots, \alpha_{m}^{2 r} A_{m}^{-r}\right) \\
& =\left(\alpha_{1}^{w_{1}} \cdots \alpha_{m}^{w_{m}}\right)^{2 r} G_{w}\left(A_{1}^{r}, \ldots, A_{m}^{r}\right)^{-1}
\end{aligned}
$$

which implies by (4.3) again that

$$
\widehat{d}_{1}\left(G_{w}\left(A_{1}^{r}, \ldots, A_{m}^{r}\right)\right) \leq\left(\alpha_{1}^{w_{1}} \cdots \alpha_{m}^{w_{m}}\right)^{r} .
$$

Therefore,

$$
\widehat{d}_{1}^{\frac{1}{r}}\left(G_{w}\left(A_{1}^{r}, \ldots, A_{m}^{r}\right)\right) \leq G_{w}\left(\widehat{d}_{1}\left(A_{1}\right), \ldots \widehat{d}_{1}\left(A_{m}\right)\right) .
$$

The remaining proof is an application of the standard antisymmetric tensor power technique (for this see Remark 4.5 below), as in the proof of [4, Theorem 3] with use of [5, Theorem 4.3].

Remark 4.5. For $k=1, \ldots, 2 n$ let $J^{(k)}:=\wedge^{k} J$, the $k$-fold antisymmetric tensor power of $J$. For any $A \in \mathbb{P}_{2 n}$, since (2.2) implies that

$$
\prod_{i=1}^{k} \widehat{d}_{i}(A)=\lambda_{i}^{1 / 2}\left(\left(\wedge^{k} A\right)^{1 / 2} J^{(k) T}\left(\wedge^{k} A\right) J^{(k)}\left(\wedge^{k} A\right)^{1 / 2}\right),
$$

the last part of the above proof can be carried out, although $J^{(k)}$ is not a $J$-matrix of size $\left(\begin{array}{c}2 n \\ k\end{array}\right)$ in the definition of the symplectic Lie group $\operatorname{Sp}\left(\left(\begin{array}{c}2 n \\ k\end{array}\right), \mathbb{R}\right)$ (see Section 2).

Proof of Theorem 4.3. Let $0<r \leq 1$. Lemma 4.4 says in particular that (4.2) holds when $\mu \in \mathcal{P}_{0}\left(\mathbb{P}_{2 n}\right)$. Now let $\mu \in \mathcal{P}^{1}\left(\mathbb{P}_{2 n}\right)$. By density, we can find a sequence $\mu_{k} \in \mathcal{P}_{0}\left(\mathbb{P}_{2 n}\right)$ converging to $\mu$ for the Wasserstein metric $\delta^{W}$. By Theorem 2.5, $\delta^{W}\left(\widehat{d}_{*} \mu_{k}, \widehat{d}_{*} \mu\right) \rightarrow 0$ as $k \rightarrow \infty$. Since $\mu \rightarrow \mu^{r}$ is a contraction from $\mathcal{P}^{1}\left(\mathbb{P}_{2 n}\right)$ into itself, $\delta^{W}\left(\mu_{k}^{r}, \mu^{r}\right) \leq \delta^{W}\left(\mu_{k}, \mu\right) \rightarrow 0$. By the fundamental contraction property,

$$
\delta\left(G\left(\mu_{k}^{r}\right), G\left(\mu^{r}\right)\right) \leq \delta^{W}\left(\mu_{k}^{r}, \mu^{r}\right) \rightarrow 0
$$

and also

$$
\delta\left(G\left(\widehat{d}_{*} \mu_{k}\right), G\left(\widehat{d}_{*} \mu\right)\right) \leq \delta^{W}\left(\widehat{d}_{*} \mu_{k}, \widehat{d}_{*} \mu\right) \rightarrow 0 .
$$


Since $\widehat{d}$ and $\widehat{d}_{*}$ are continuous, we have $\widehat{d}\left(G\left(\mu_{k}^{r}\right)\right) \rightarrow \widehat{d}\left(G\left(\mu^{r}\right)\right)$ as well as $G\left(\widehat{d}_{*} \mu_{k}\right) \rightarrow$ $G\left(\widehat{d}_{*} \mu\right)$ in $\mathbb{R}_{+}^{2 n}$. By Lemma 4.4 we have $\widehat{d}^{\frac{1}{r}}\left(G\left(\mu_{k}^{r}\right)\right) \underset{\log }{\prec} G\left(\widehat{d}_{*} \mu_{k}\right)$. Hence letting $k \rightarrow \infty$ gives $\widehat{d}^{\frac{1}{r}}\left(G\left(\mu^{r}\right)\right) \underset{\log }{\prec} G\left(\widehat{d}_{*} \mu\right)$.

Remark 4.6. Let $0<r<1$. Compared with the log-majorizations in (4.1) one may think of the following, where $\mu \in \mathcal{P}^{1}\left(\mathbb{P}_{2 n}\right), A, B \in \mathbb{P}_{2 n}$ and $0<t<1$ :

(a) $\widehat{d}\left(G\left(\mu^{r}\right)^{\frac{1}{r}}\right) \underset{\log }{\prec} G\left(\widehat{d}_{*} \mu\right)$ ? In particular, $\widehat{d}\left(\left(A^{r} \#_{t} B^{r}\right)^{\frac{1}{r}}\right) \underset{\log }{\prec} \widehat{d}^{1-t}(A) \widehat{d}^{t}(B)$ ?

(b) $\widehat{d}\left(G(\mu)^{r}\right) \underset{\log }{\prec} \widehat{d}\left(G\left(\mu^{r}\right)\right)$ ? In particular, $\widehat{d}\left(\left(A \#_{t} B\right)^{r}\right) \underset{\log }{\prec} \widehat{d}\left(A^{r} \#_{t} B^{r}\right)$ ?

(c) $\widehat{d}\left(\exp \int_{\mathbb{P}_{2 n}} \log X d \mu(X)\right) \underset{\log }{\prec} G\left(\widehat{d}_{*} \mu\right)$ ?

When $n=1$, since $\widehat{d}(X)=\left(\operatorname{det}^{\frac{1}{2}}(X)\right.$, $\left.\operatorname{det}^{\frac{1}{2}}(X)\right)$ for any $X \in \mathbb{P}_{2}$, the above are all trivial as both sides of each of $(\mathrm{a})-(\mathrm{c})$ are equal. However, when $n \geq 2$, it is rather difficult for us to expect that the log-majorizations in (a)-(c) hold true, while we have no explicit counterexamples.

We have directly the following general version, which provides the proof of the main result (Theorem 1.1). Indeed, $\varphi_{*} \mathbf{P} \in \mathcal{P}^{1}\left(\mathbb{P}_{n}\right)$ for every $\varphi \in L^{1}\left(\Omega ; \mathbb{P}_{n}\right)$, where $(\Omega, \mathbf{P})$ is a probability space, and then by Theorem 4.1,

$$
\lambda\left(G\left(\varphi_{*} \mathbf{P}\right)\right) \underset{\log }{\prec} G\left(\lambda_{*}\left(\varphi_{*} \mathbf{P}\right)\right)=G\left((\lambda \circ \varphi)_{*} \mathbf{P}\right),
$$

and similarly for the case of the symplectic eigenvalues when $\varphi \in L^{1}\left(\Omega ; \mathbb{P}_{2 n}\right)$.

Theorem 4.7. Let $(\Omega, \mathbf{P})$ be a probability space. Then for every $\varphi \in L^{1}\left(\Omega ; \mathbb{P}_{n}\right)$, that is, $\varphi: \Omega \rightarrow \mathbb{P}_{n}$ satisfying $\int_{\Omega} \delta(\varphi(\omega), X) d \mathbf{P}(\omega)<\infty$ for some $X \in \mathbb{P}_{n}$,

$$
\lambda\left(G\left(\varphi_{*} \mathbf{P}\right)\right) \underset{\log }{\prec} G\left((\lambda \circ \varphi)_{*} \mathbf{P}\right) .
$$

Moreover, for every $\varphi \in L^{1}\left(\Omega ; \mathbb{P}_{2 n}\right)$,

$$
\widehat{d}\left(G\left(\varphi_{*} \mathbf{P}\right)\right) \underset{\log }{\prec} G\left((\widehat{d} \circ \varphi)_{*} \mathbf{P}\right) .
$$

More precisely we have from (4.1), 
Corollary 4.8. For every $\varphi \in L^{1}\left(\Omega ; \mathbb{P}_{n}\right)$,

$$
\begin{aligned}
\lambda\left(\int_{\Omega}^{(G)} \varphi(\omega) d \mathbf{P}(\omega)\right) & \underset{\log }{\prec} \lambda^{\frac{1}{r}}\left(\int_{\Omega}^{(G)} \varphi(\omega)^{r} d \mathbf{P}(\omega)\right) \\
& \prec \lambda\left(\operatorname{loxp} \int_{\Omega} \log \varphi(\omega) d \mathbf{P}(\omega)\right) \\
& \underset{\log }{\prec} \int_{\Omega}^{(G)} \lambda(\varphi(\omega)) d \mathbf{P}(\omega) .
\end{aligned}
$$

5. Log-MAJorizATIONS FOR MULtiple PROBABILITy MEASURES

There is a natural notion of multivariate "geometric" mean of integrable probability Borel measures [9]. The Cartan mean of $m$ positive definite matrices $G: \mathbb{P}_{n}^{m} \rightarrow \mathbb{P}_{n}$ is Lipschitz from the fundamental contraction property and hence induces a Lipschitz map $\Lambda:\left(\mathcal{P}^{1}\left(\mathbb{P}_{n}\right)\right)^{m} \rightarrow \mathcal{P}^{1}\left(\mathbb{P}_{n}\right)$ defined by

$$
\Lambda\left(\mu_{1}, \ldots, \mu_{m}\right):=G_{*}\left(\mu_{1} \times \cdots \times \mu_{m}\right) \in \mathcal{P}^{1}\left(\mathbb{P}_{n}\right) .
$$

Note that $\Lambda(\mu)=\mu$ for $m=1$. By our log-majorization in Theorem 4.1,

$$
\lambda\left(G\left(\Lambda\left(\mu_{1}, \ldots, \mu_{m}\right)\right)\right) \underset{\log }{\prec} G\left(\lambda_{*} \Lambda\left(\mu_{1}, \ldots, \mu_{m}\right)\right)=G\left((\lambda \circ G)_{*}\left(\mu_{1} \times \cdots \times \mu_{m}\right)\right) .
$$

However, from $\lambda_{*} \mu_{j} \in \mathcal{P}^{1}\left(\mathbb{R}_{+}^{n}\right)$,

$$
\Lambda\left(\lambda_{*} \mu_{1}, \ldots, \lambda_{*} \mu_{m}\right):=G_{*}\left(\lambda_{*} \mu_{1} \times \cdots \times \lambda_{*} \mu_{n}\right) \in \mathcal{P}^{1}\left(\mathbb{R}_{+}^{n}\right)
$$

and $G\left(\Lambda\left(\lambda_{*} \mu_{1}, \ldots, \lambda_{*} \mu_{m}\right)\right) \in \mathbb{R}_{+}^{n}$. Between this and both sides of (5.1) we have the following log-majorizations.

Theorem 5.1. For every $\mu_{1}, \ldots, \mu_{m} \in \mathcal{P}^{1}\left(\mathbb{P}_{n}\right)$,

$$
\lambda\left(G\left(\Lambda\left(\mu_{1}, \ldots, \mu_{m}\right)\right)\right) \underset{\log }{\prec} G\left(\lambda_{*} \Lambda\left(\mu_{1}, \ldots, \mu_{m}\right)\right) \underset{\log }{\prec} G\left(\Lambda\left(\lambda_{*} \mu_{1}, \ldots, \lambda_{*} \mu_{m}\right)\right) .
$$

Proof. It remains to prove the second log-majorization. As mentioned above the theorem, note that $G: \mathbb{P}_{n}^{m} \rightarrow \mathbb{P}_{n}$ and $\Lambda:\left(\mathcal{P}^{1}\left(\mathbb{P}_{n}\right)\right)^{m} \rightarrow \mathcal{P}^{1}\left(\mathbb{P}_{n}\right)$ are Lipschitz continuous, as well as so are $\lambda: \mathbb{P}_{n} \rightarrow \mathbb{R}_{+}^{n}$ and $\lambda_{*}: \mathcal{P}^{1}\left(\mathbb{P}_{n}\right) \rightarrow \mathcal{P}^{1}\left(\mathbb{R}_{+}^{n}\right)$ (see Example 2.3). So it suffices by continuity to prove the assertion for $\mu_{1}, \ldots, \mu_{n} \in \mathcal{P}_{0}\left(\mathbb{P}_{n}\right)$. Let $\mu_{j}=\left(1 / k_{j}\right) \sum_{i=1}^{k_{j}} \delta_{A_{j i}}$ for $j=1, \ldots, m$. Then

$$
\Lambda\left(\mu_{1}, \ldots, \mu_{m}\right)=\frac{1}{k_{1} \cdots k_{m}} \sum_{i_{1}, \ldots, i_{m}} \delta_{G\left(A_{1 i_{1}, \ldots, A_{m i_{m}}}\right)}
$$


where the sum is taken over all $i_{j}=1, \ldots, k_{j}$ and $j=1, \ldots, m$. We hence have from (3.3)

$$
\lambda_{*} \Lambda\left(\mu_{1}, \ldots, \mu_{m}\right)=\frac{1}{k_{1} \cdots k_{m}} \sum_{i_{1}, \ldots, i_{m}} \delta_{\lambda\left(G\left(A_{1 i_{1}}, \ldots, A_{m i_{m}}\right)\right)}
$$

so that

$$
G\left(\lambda_{*} \Lambda\left(\mu_{1}, \ldots, \mu_{m}\right)\right)=G\left(\lambda\left(G\left(A_{1 i_{1}}, \ldots, A_{m i_{m}}\right)\right): i_{1}, \ldots, i_{m}\right)
$$

where the right-hand side of (5.3) is the geometric mean as an element of $\left(\mathbb{R}_{+}^{n}\right)^{k_{1} \cdots k_{m}}$. On the other hand, since $\lambda_{*} \mu_{j}=\left(1 / k_{j}\right) \sum_{i=1}^{k_{j}} \delta_{\lambda\left(A_{j i}\right)}$, we have

$$
G\left(\Lambda\left(\lambda_{*} \mu_{1}, \ldots, \lambda_{*} \mu_{m}\right)\right)=G\left(G\left(\lambda\left(A_{1 i_{1}}\right), \ldots, \lambda\left(A_{m i_{m}}\right)\right): i_{1}, \ldots, i_{m}\right) .
$$

By the log-majorization in [5, (30)] (also Theorem 4.1),

$$
\lambda\left(G\left(A_{1 i_{1}}, \ldots, A_{m i_{m}}\right)\right) \underset{\log }{\prec} G\left(\lambda\left(A_{1 i_{1}}\right), \ldots, \lambda\left(A_{m i_{m}}\right)\right)
$$

for all $i_{1}, \ldots, i_{m}$. Combining this with (5.3) and (5.4) we easily see the second logmajorization asserted.

When $m=1$, since $\lambda(G(\Lambda(\mu)))=\lambda(G(\mu))$ and $G\left(\lambda_{*} \Lambda(\mu)\right)=G\left(\Lambda\left(\lambda_{*} \mu\right)\right)=G\left(\lambda_{*} \mu\right)$, (5.2) is included in (4.1). When $\mu_{j}=\delta_{A_{j}}$ for $j=1, \ldots, m$, since the first two terms of (5.2) are $\lambda\left(G\left(A_{1}, \ldots, A_{m}\right)\right)$ from $\Lambda\left(\delta_{A_{1}}, \ldots, \delta_{A_{m}}\right)=\delta_{G\left(A_{1}, \ldots, A_{m}\right)}$ and the last term is $G\left(\lambda\left(A_{1}\right), \ldots, \lambda\left(A_{m}\right)\right)$ by (3.3), (5.2) reduces to (1.2).

For $\mu_{1}, \ldots, \mu_{m} \in \mathcal{P}^{1}\left(\mathbb{P}_{2 n}\right)$ the log-majorization in Theorem 4.3 gives

$$
\widehat{d}\left(G\left(\Lambda\left(\mu_{1}, \ldots, \mu_{m}\right)\right)\right) \underset{\log }{\prec} G\left(\widehat{d}_{*} \Lambda\left(\mu_{1}, \ldots, \mu_{m}\right)\right) .
$$

The proof of the second log-majorization of (5.5) is similar to that of (5.2) above by using [4, (20)] (also Theorem 4.3) in place of [5, (30)].

Theorem 5.2. For every $\mu_{1}, \ldots, \mu_{m} \in \mathcal{P}^{1}\left(\mathbb{P}_{2 n}\right)$,

$$
\widehat{d}\left(G\left(\Lambda\left(\mu_{1}, \ldots, \mu_{m}\right)\right)\right) \underset{\log }{\prec} G\left(\widehat{d}_{*} \Lambda\left(\mu_{1}, \ldots, \mu_{m}\right)\right) \underset{\log }{\prec} G\left(\Lambda\left(\widehat{d}_{*} \mu_{1}, \ldots, \widehat{d}_{*} \mu_{m}\right)\right) .
$$




\section{ACKnowledgements}

The work of F. Hiai was supported in part by Grant-in-Aid for Scientific Research (C)17K05266. The work of Y. Lim was supported by the National Research Foundation of Korea (NRF) grant funded by the Korea government (MEST) No.NRF2015R1A3A2031159 and 2016R1A5A1008055.

\section{REFERENCES}

[1] Arvind, B. Dutta, N. Mukunda and R. Simon, The real symplectic groups in quantum mechanics and optics, Pramana 45 (1995), 471-497.

[2] R. Bhatia, Matrix Analysis, Springer-Verlag, New York, 1996.

[3] R. Bhatia, Positive definite matrices, Princeton Series in Applied Mathematics, Princeton University Press, Princeton, NJ, 2007.

[4] R. Bhatia and T. Jain, On symplectic eigenvalues of positive definite matrices, J. Math. Phys. 56 (2015), 112201.

[5] R. Bhatia and R. Karandikar, Monotonicity of the matrix geometric mean, Math. Ann. 353 (2012), 1453-1467.

[6] G. Corach, H. Porta, and L. Recht, Convexity of the geodesic distance on spaces of positive operators, Illinois J. Math. 38 (1994), 87-94.

[7] J. Eisert, T. Tye, T. Rudolph, and B. C. Sanders, Gaussian quantum marginal problem, Commun. Math. Phys. 280 (2008), 1453-1467.

[8] F. Hiai, Matrix Analysis: Matrix Monotone Functions, Matrix Means, and Majorization, Interdisciplinary Information Sciences 16 (2010), 139-248.

[9] F. Hiai, J. Lawson and Y. Lim, The Stochastic order of probability measures on ordered metric spaces, preprint.

[10] F. Hiai and Y. Lim, Log-majorization and Lie-Trotter formula for the Cartan barycenter on probability measure spaces, J. Math. Anal. Appl. 453 (2017), 195-211.

[11] F. Hiai and Y. Lim, Geometric mean flows and the Cartan barycenter on the Wasserstein space over positive definite matrices, Linear Algebra Appl. 533 (2017), 118-131.

[12] J. Lawson and Y. Lim, Monotonic properties of the least squares mean, Math. Ann. 351 (2011), 267-279.

[13] J. Lawson and Y. Lim, Contractive barycentric maps, J. Operator Theory 77 (2017), 87-107.

[14] R. D. Nussbaum, Finsler structures for the part metric and Hilbert's projective metric and applications to ordinary differential equations, Differential and Integral Equations 7 (1994), 1649-1707. 
[15] K. R. Parthasarathy, The symmetry group of Gaussian states in $L^{2}\left(\mathbb{R}^{n}\right)$, in Prokhorov and Contemporary Probability, A. N. Shiryaev, S. R. S. Varadhan and E. L. Presman (eds.), Springer Proceedings in Mathematics and Statistics 33 (2013), 349-369.

[16] K.-T. Sturm, Probability measures on metric spaces of nonpositive curvature. Heat kernels and analysis on manifolds, graphs, and metric spaces (Paris, 2002), 357-390, Contemp. Math. 338, Amer. Math. Soc., Providence, RI, 2003.

Tohoku University (Emeritus), Hakusan 3-8-16-303, Abiko 270-1154, Japan

E-mail address: fumio.hiai@gmail.com

Department of Mathematics, Sungkyunkwan University, Suwon 440-746, Korea

E-mail address: ylim@skku.edu 